\title{
Logical Structure of the Immunoregulatory Compartment
}

\author{
N.A. Mitchison
}

The immune system as we know it in vertebrates is divided into a number of compartments, each of which contains a more or less complete set of lymphocytes with diverse receptors. Among them, by far the largest single compartment is devoted to immunoregulation and comprises the $\mathrm{CD} 4^{+}$, class II MHC-restricted set of $\mathrm{T}$ cells. Our invertebrate progenitors have molecules that are distantly homologous to antibody, but in this ancestral form they are believed to function in cell adhesion rather than defence, and are not diversified. At a stage in the evolution of vertebrates they developed a mechanism of diversification, and at that point must have been distributed on a single set of lymphocytes. This non-compartmentalized stage has not been found in any living vertebrates such as the primitive jawless fish. The rapid replacement of this missing link tells us that a compartmentalized immune system must provide significant evolutionary benefits.

The existence of a regulatory compartment permits:

1. Better antibodies to be made, by means of hypermutation

2. Coordination of the immunological attack on complex antigens, by means of epitope linkage

3. Control of hypersensitivity, by means of immunosuppression

Among these benefits, the third is the odd one out as it is needed only after the

Imperial Cancer Research Fund, Tumour Immunology Unit, University College London, Department of Biology, Medawar Building, Gower Street, London, WC1E 6BT, UK regulatory compartment has evolved; it corrects some of the problems that then arise as a result of overactivity within that compartment.

Each of these benefits needs further explanation. The first, that of hypermutation, is linked logically to the mechanism that the immune system has developed for avoiding reactivity with self. Tolerance of self, it is now generally agreed, results from deletion of self-reactive lymphocytes during development. This proposition needs minor qualification, in so far as mechanisms of suppression may supplement clonal deletion, but experience with unmanipulated systems (such as my group's work on F liver protein as an antigen in mice [1]) has underlined the primary role of deletion. If we accept this proposition, then it is also clear that hypermutation cannot be allowed to occur within the set of lymphocytes that is responsible for selftolerance. Rajewsky's current estimate of the hypermutation rate in memory Bcells is $\sim 10^{-2}$ /base pair per cell division; a rate as high as that would surely fill in any holes in the repertoire created by clonal deletion, and that would lead on to autoimmune disease. What actually happens is that the essential clonal deletion occurs only within the regulatory compartment (among helper T-cells), while hypermutation is confined to $\mathrm{B}$ cells, i.e., within one of the effector compartments. Clonal deletion may or may not also occur within the B-cell compartment, but that seems to be an optional extra that varies from one self macromolecule to another, depending mainly on concentration within body fluids. All this was known prior to the discovery of hyper- 
mutation; the real step forward is to understand that T-cell tolerance and hypermutation in B cells fit together logically, so that the two sets of observations mutually reinforce one another.

The second benefit, of epitope linkage, is of special interest because it has recently been discovered to work through two rather different mechanisms, and thus provides the first logical explanation of why $T$ and $B$ cells follow different traffic patterns within the immune system. Overall, linkage coordinates immunological attack in the following way: a regulatory lymphocyte (a helper $\mathrm{T}$ cell) recognizes an epitope (in effect a regulatory epitope) of a complex antigen, and then selectively activates effector lymphocytes (B cells or cytolytic $T$ cells) that recognize other epitopes (effector epitopes) of the same antigen. In much the same way a suppressor regulatory cell (a suppressor $T$ cell) can also selectively downregulate other cells (principally helper $T$ cells) that recognize the same antigen. For both $T$ and $B$ cells the selective activation works via short-range lymphokines that are not antigen specific, and so the linkage depends exclusively on the regulatory and effector cells being brought into juxtaposition. There, however, the similarity stops. The $\mathrm{T}$ to $\mathrm{B}$ interaction depends on the formation of two-cell-type clusters, in which a $B$ cell binds directly to $T$ cells. The T-to-T interaction (helper to cytolytic $T$ cell) depends rather on three-celltype clusters, in which the two types of lymphocytes bind to a common antigenpresenting cell. Two crucial pieces of evidence establish that three-cell-type clusters do in fact form under physiological conditions in vivo [2]: (a) the two types of lymphocyte need not make a cognate interaction (in contrast to the T-B interaction, where such a requirement applies), and (b) with large numbers of antigenic particles (i.e. when each antigen-presenting cell can be calculated to pick up several particles) epitope linkage no longer operates (in contrast, again, to the T-B interaction, which saturates, if at all, only at much higher particle numbers).
Thus the immune system requires two quite different types of antigen-presenting cell. One, for B cells, must retain antigen in a conformationally intact form (most B cells recognize conformation epitopes); it must do so long term, in order to provide time for hypermutation to operate; and its dendrites need to be spaced together in a network dense enough to maximize the chances of contact with migrating $B$ cells. The other, for $T$ cells, must cleave antigen into a form able to bind to major histocompatibility complex (MHC) molecules; it need not retain antigen long term, for $\mathrm{T}$ cells do not hypermutate and their response plateaus early; and the presenting cells with their dendrites need to be spaced far enough apart to prevent the three-cell-type clusters, each with its own presenting cell, from interacting with one another and thus interfering with epitope linkage [3].

If one looks at the T-cell and B-cell area of a lymph node, two quite different (and possibly unrelated) types of antigenpresenting cell are evident in the two areas. Among $T$ cells are to be found interdigitating dendritic cells, and among B cells follicular dendritic cells. Each has the appropriate combination of the three contrasting properties that have just been described. Furthermore the interdigitating dendritic cell, as well as being able to process antigen so that it associates with MHC molecule, is known also to bind T cells spontaneously in the absence of antigen and to stimulate them effectively in its presence. It is natural to conclude that the segregation of a lymph node into these two areas depends primarily on the need for the two types of antigen-presenting cell, and indeed that once the dendritic cells have sorted themselves out everything else in a lymph node will follow automatically as a consequence of selective aggregation of lymphocytes.

This account of lymph node structure is far from complete. It leaves unexplained the initial phase of the immune response, prior to localization of antigen on follicular dendritic cells in the form of antigen-antibody complexes; this early 
phase is still poorly understood. In addition, there is probably more to the structure of a follicle than simply aggregation of B cells around their antigen, for the germinal centre, the outer follicle, and the marginal zone around it are carefully arranged in a way that still require explanation.

The third benefit, of immunosuppression, raises the controversial issue of the suppressor $\mathrm{T}$ cell. Opinions among immunologists vary from those who regard this cell as playing a fundamental role in regulation of the immune response, to those who regard it as no more than an illusion. My own opinion, expressed in several recent and forthcoming reviews [4-7], lies somewhere between these two poles. I think it likely that a suppressor mechanism does operate, and that it has evolved primarily as a measure to counteract that threat of hypersensitivity. Over the course of evolution it is likely that the main threat of hypersensitivity has come from chronic infection: witness the extent to which chronic parasitic infection in the third world is usually well tolerated, except when it generates hypersensitivity. This line of thought finds support from studies in immunogenetics. On present evidence that MHC class II genes that mainly mediate suppression are HLA-DQ in man and $\mathrm{H}-2 \mathrm{E}$ in the mouse; as these are not homologous, this function must have flipped from one locus to another during mammalian evolution of the mammals. Furthermore mice, and possible rats too, fairly often lose expression of their suppression-mediating $\mathrm{MHC}$ class genes, as though the selective pressure of hypersentivity is diminished in these short-lived species.

As for the mechanism of suppression, the central questions remain unanswered pending the full deployment of molecular biology in this area. It is entirely possible that the phenomena of suppression can be accounted for by the known properties of suppression-mediating T cells: specialized restriction, surface markers such as CD45R, lymphokine-secretion profile, and high connectivity. Alternatively, these composite properties may eventually lead us to a set of T cells that have their own unique molecular mechanisms, such as a new set of receptors. The question is still open, and must surely occupy a high position on the agenda of contemporary immunology.

\section{References}

1. Griffiths JA, Mitchison NA, Nardi N, Oliveira DBG (1987) F protein. In: Sercarz $\mathrm{E}$, Berzofsky $\mathrm{J}$ (eds) Immunogenicity of protein antigens: repertoire and regulation, Vol II. CRC, Boca Raton, pp 35-40

2. Mitchison NA, O'Malley C (1987) Three cell type clusters of T-cells with antigen presenting cells best explain the epitope linkage and non-cognate requirements of the in vivo cytolytic response. Eur J Immunol 17:579-583

3. Dexter $M$, Marvel J, Merkenschlager $M$, Mitchison NA, Oliveira D, O'Malley C, Smith L, Terry L, Timms E (1987) Progress in $\mathrm{T}$ cell biology. Immunol Lett 16:171178

4. Mitchison NA, Oliveira DBG (1986) Chronic infection as a major force in the evolution of the suppressor $\mathrm{T}$ cell system. Parasitol Today 2:312-313

5. Mitchison NA, Oliveira DBG (1986) Epirestriction and a specialised subset of $T$ helper cells are key factors in the regulation of T suppressor cells. In: Cinader B, Miller RG (eds) Progress in immunology, vol VI. Academic Press, London, pp 326-334

6. Marvel J, Mitchison NA, Oliveira DBG, O'Malley C (1987) The split within the CD4 (helper) T-cell subset, and its implications for immunopathology. Mem Inst Oswaldo Cruz [Suppl I] 82:260-273

7. Oliveira DBG, Mitchison NA (1989) Immune suppression genes. Clin Exp Immunol 75:167-177 